\title{
RSS-Based Localization in Wireless Sensor Networks Using Convex Relaxation: Noncooperative and Cooperative Schemes
}

\author{
Slavisa Tomic, Marko Beko, and Rui Dinis, Member, IEEE
}

\begin{abstract}
In this paper, we propose new approaches based on convex optimization to address the received signal strength (RSS)-based noncooperative and cooperative localization problems in wireless sensor networks (WSNs). By using an array of passive anchor nodes, we collect the noisy RSS measurements from radiating source nodes in WSNs, which we use to estimate the source positions. We derive the maximum likelihood (ML) estimator, since the ML-based solutions have particular importance due to their asymptotically optimal performance. However, the ML estimator requires the minimization of a nonconvex objective function that may have multiple local optima, thus making the search for the globally optimal solution hard. To overcome this difficulty, we derive a new nonconvex estimator, which tightly approximates the ML estimator for small noise. Then, the new estimator is relaxed by applying efficient convex relaxations that are based on second-order cone programming and semidefinite programming in the case of noncooperative and cooperative localization, respectively, for both cases of known and unknown source transmit power. We also show that our approaches work well in the case when the source transmit power and the path loss exponent are simultaneously unknown at the anchor nodes. Moreover, we show that the generalization of the new approaches for the localization problem in indoor environments is straightforward. Simulation results show that the proposed approaches significantly improve the localization accuracy, reducing the estimation error between $15 \%$ and $20 \%$ on average, compared with the existing approaches.
\end{abstract}

Index Terms-Centralized localization, cooperative localization, noncooperative localization, received signal strength (RSS),

Manuscript received December 17, 2013; revised April 23, 2014 and June 19, 2014; accepted June 26, 2014. Date of publication July 1, 2014; date of current version May 12, 2015. This work was supported in part by the Fundação para a Ciência e a Tecnologia under Projects PEst-OE/EEI/ UI0066/2014, EXPL/EEI-TEL/0969/2013-MANY2COMWIN and EXPL/ EEI-TEL/1582/2013-GLANC, PEst-OE/EEI/LA0008/2013 (IT pluriannual founding and HETNET), PEst-OE/EEI/UI0066/2011 (UNINOVA pluriannual founding), EnAcoMIMOCo EXPL/EEI-TEL/2408/2013, and ADIN PTDC/ EEI-TEL/2990/2012, and Grant SFRH/BD/91126/2012 and the Ciência 2008 Post-Doctoral Research grant. This paper was presented in part at the IEEE Workshop on Signal Processing Advances in Wireless Communications (SPAWC 2013) Darmstadt, Germany, June 16-19, 2013. The review of this paper was coordinated by Prof. G. Mao.

$\mathrm{S}$. Tomic is with the Institute for Systems and Robotics (ISR), Instituto Superior Técnico, Universidade de Lisboa, 1049-001 Lisbon, Portugal (e-mail: stomic@isr.ist.utl.pt).

M. Beko is with the Universidade Lusófona de Humanidades e Tecnologias, 1749-024 Lisbon, Portugal, and also with UNINOVA, 2829-516 Caparica, Portugal (e-mail: beko.marko@ulusofona.pt; mbeko@uninova.pt).

R. Dinis is with the Instituto de Telecomunicações, 1049-001 Lisbon, Portugal; with the Centre of Technology and Systems (CTS), UNINOVA, 2829-516 Caparica, Portugal; and also with the Departamento de Engenharia Electrotécnica, Faculdade de Ciências e Tecnologia (FCT), Universidade Nova de Lisboa, 2829-516 Caparica, Portugal (e-mail: rdinis@ fct.unl.pt).

Color versions of one or more of the figures in this paper are available online at http://ieeexplore.ieee.org.

Digital Object Identifier 10.1109/TVT.2014.2334397 second-order cone programming (SOCP) problem, semidefinite programming (SDP) problem, wireless localization, wireless sensor network (WSN).

\section{INTRODUCTION}

W IRELESS SENSOR networks (WSNs) comprise a number of sensor nodes, which can, in general, be classified as anchor and source (target) nodes [1]. The locations of the anchor nodes are known, whereas the locations of the source nodes are yet to be determined. WSNs have application in various areas such as target tracking, intrusion detection, energy-efficient routing, monitoring, underground, deep water, outer space explorations, etc. [2]. In WSNs, sensor nodes are deployed over a monitored region to acquire some physical data about the environment, such as temperature, pressure, humidity, wind speed, etc. The collected information, together with the object's position information, enables us to develop intelligent systems. Such systems offer improved safety and efficiency in everyday life, since each individual device in the network can respond faster and better to the changes in the environment (e.g., location-aware vehicles and asset management in warehouses) [3]. The idea of wireless positioning was initially conceived for cellular networks, since it invokes many innovative applications and services for its users. Nowadays, rapid increase in heterogeneous smart devices (mobile phones and tablets), which offer self-sustained applications and seamless interfaces to various wireless networks, is pushing the role of the location information to become a crucial component for mobile context-aware applications [1].

Location information in WSNs is usually obtained by rangebased or range-free measurements. In this paper, we focus only on the former, since they provide higher estimation accuracy in general. In the range-based localization process, the main concern is to accomplish good estimation accuracy from inaccurate position-bearing measurements collected inside the network. To obtain the information of interest, it is necessary to enable node communication, which can be noncooperative or cooperative. The former approach allows source nodes to communicate only with the anchor nodes, whereas the latter approach allows source nodes to communicate with all nodes inside their communication range, whether they are anchor or source nodes [1]. Algorithmically, both approaches can be executed in a distributed (self-positioning) or centralized (network-centric positioning) mode. Although the distributed approach has, in general, low complexity and high scalability, 
it is sensitive to error propagation and may require long convergence time. Therefore, in this paper, we concentrate only on the centralized approach. To establish an accurate estimate of the source position, the processor must have prior knowledge about the anchor nodes' positions. In cellular networks, the base stations can be seen as anchor nodes, and the mobile stations can be seen as source nodes.

Depending on the available hardware, current distancebased localization techniques exploit different measurements of the radio signal transmitted between nodes [received signal strength (RSS), time-difference-of-arrival (TDOA), time-ofarrival (TOA), roundtrip-time (RTT), or angle-of-arrival (AOA) measurements]. The tradeoff between the localization accuracy and the implementation complexity of each technique is a very important factor when deciding which method to employ. For example, localization based on TOA or TDOA [including the Global Positioning System (GPS)] gives high estimation accuracy, but it requires a very complex process of timing and synchronization, thus making the localization cost expensive [4]. Although less accurate than the localization using TOA, TDOA, or AOA information, localization based on the RSS measurements requires no specialized hardware, less processing, and communication (and, consequently, lower energy), thus making it an attractive low-cost solution for the localization problem [2], [5]. Another attractive low-cost approach might be exploiting RTT measurements, which are easily obtained in wireless local area network (WLAN) systems by using a simple device such as a printed circuit board [6]. Although RTT systems circumvent the problem of clock synchronization between nodes, the major drawback of this approach is the need for double signal transmission to perform a single measurement [7]. Recently, hybrid methods that fuse two measurements of the radio signal (e.g., RSS-RTT) attracted considerable attention in the research society [6], [8], [9]. These methods try to improve the estimation accuracy in node positions by exploiting the benefits of the combined measurements, together with minimizing their drawbacks. In this paper, we focus on providing a good localization accuracy by using RSS measurements exclusively.

\section{A. Related Work}

Source localization based on the RSS measurements has recently attracted much attention in the wireless communications community [10]-[18]. The most popular estimator used in practice is the maximum likelihood (ML) estimator, since it is asymptotically efficient (for large enough data records) [19]. However, solving the ML estimator of the RSS-based localization problem is a very difficult task, because it is highly nonlinear and noncovex [5]; hence, it may have multiple local optima. In this case, search for the globally optimal solution is very hard via iterative algorithms, since they may converge to a local minimum or a saddle point resulting in a large estimation error. To overcome this difficulty and possibly provide a good initial point (close to the global minimum) for the iterative algorithms, approaches such as grid search methods, linear estimators, and convex relaxation techniques have been introduced to address the ML problem [10]-[18].
The grid search methods are time consuming and require a huge amount of memory when the number of unknown parameters is too large. Linear estimators are very efficient in the sense of time consumption, but they are derived based on many approximations that may affect their performance, particularly in the case when the noise is large [14]. In convex relaxation techniques such as those in [12]-[18], the difficulties in the ML problem are overcome by transforming the original nonconvex and nonlinear problem into a convex problem. The advantage of this approach is that convergence to the globally optimal solution is guaranteed. However, due to the use of relaxation techniques, the solution of a convex problem does not necessarily correspond to the solution of the original ML problem [20].

In [10], different weighting schemes for the multidimensional scaling (MDS) formulation were presented and compared. It was shown that the solution of the MDS can be used as the initial value for iterative algorithms, which then converge faster and attain higher accuracy when compared with random initial values. Convex semidefinite programming (SDP) estimators were proposed in [12] to address the nonconvexity of the ML estimator, for both noncooperative and cooperative localization problems with known source transmit power, i.e., $P_{T}$. Ouyang et al. in [12] reformulated the localization problem by eliminating the logarithms in the ML formulation and approaching the localization problem as a minimax optimization problem, which is then relaxed as SDP. Although the approach described in [12] provides good estimation results, particularly for the case of cooperative localization, it has high computational complexity, which might restrict its application in large-scale WSNs. In [13], the RSS-based localization problem for known $P_{T}$ was formulated as the weighted least squares (WLS) problem, based on the unscented transformation (UT). It was shown that for the cooperative localization, the WLS formulation can be relaxed as a mixed semidefinite and second-order cone programming problem (SD/SOCP), whereas for the noncooperative localization, the WLS problem can be solved by the bisection method. In [16], Wang et al. addressed the noncooperative RSS localization problem for the case of unknown $P_{T}$ and the path loss exponent (PLE). For the case of unknown $P_{T}$, based on the UT, a WLS formulation of the problem is derived, which was solved by the bisection method. When both $P_{T}$ and PLE are not known, an alternating estimation procedure is introduced. However, both [13] and [16] have the assumption of perfect knowledge of the noise standard deviation (STD). This might not be the case in practice, particularly in low-cost systems such as RSS where calibration is avoided due to maintaining low system costs [2], [5]. In [18], Vaghefi et al. addressed the RSS cooperative localization problem for unknown $P_{T}$. The case where the source nodes have different $P_{T}$ (e.g., due to different antenna gains) was considered in [18]. The authors solved the localization problem by applying an SDP relaxation technique and converting the original ML problem into a convex problem. Furthermore, in [18], the authors examined the effect of imperfect knowledge of the PLE on the performance of the SDP algorithm and used an iterative procedure to solve the problem when $P_{T}$ and PLE are simultaneously unknown. 


\section{B. Contributions}

In this paper, the RSS-based source localization problem for both noncooperative and cooperative scenarios is considered. Instead of solving the ML problem, which is highly nonconvex and computationally exhausting to solve globally, we propose a suboptimal approach that provides an efficient solution. Hence, we introduce a new nonconvex least squares (LS) estimator that tightly approximates the ML estimator for small noise. This estimator represents a smoother and simpler localization problem in comparison to the ML problem. Applying appropriate convex relaxations to the derived nonconvex estimator, novel SOCP and novel mixed SDP/SOCP estimators are proposed for noncooperative and cooperative localization cases, respectively.

The proposed approach offers an advantage over the existing approaches as it allows straightforward adaptation to different scenarios of the RSS localization problem, thereby significantly reducing the estimation error. In both noncooperative and cooperative scenarios, we first consider the simplest case of the localization problem where $P_{T}$ is known at the anchor nodes. Next, we consider a more realistic scenario in which we assume that $P_{T}$ is an unknown parameter, and we generalize our approaches for this setting. Finally, we investigate the most challenging scenario of the localization problem when $P_{T}$ and PLE are simultaneously unknown at the anchor nodes. In this case, for the noncooperative localization, we apply an iterative procedure based on the proposed SOCP method to estimate all unknown parameters. We also provide details about the computational complexity of the considered algorithms.

In contrast to [12] and [13] where the authors considered the localization problem for the case when $P_{T}$ is known, here, we address a more challenging scenario when both $P_{T}$ and PLE are not known. In [13] and [16], Wang and Yang and Wang et al., respectively, assumed that accurate knowledge of the noise STD is available, which might not be a valid assumption in some practical scenarios. Hence, we consider a more realistic scenario in which the noise STD is not available. In contrast to [18] where an SDP estimator is derived for the case of unknown $P_{T}$, we derive our estimators by using SOCP relaxation for the noncooperative case and mixed SDP/SOCP relaxation for the cooperative case.

The remainder of this paper is structured as follows. In Section II, the RSS measurement model for locating a single source node is introduced, the source localization problem is formulated for the case of known source transmit power, and the development of the proposed SOCP estimator is presented. We then extend this approach for the case where $P_{T}$, and $P_{T}$ and PLE are simultaneously unknown. Section III introduces the RSS measurement model for the cooperative localization where multiple source nodes are simultaneously located. We give the formulation of the cooperative localization problem and provide details on the development of the proposed SDP estimators for both cases of known and unknown source transmit power. The complexity analysis is summarized in Section IV. In Section V, we provide both the complexity and simulation results to compare the performance of the newly proposed estimators with the existing estimators. Finally, in Section VI, we summarize the main conclusions.

\section{NONCOOPERATIVE LOCALIZATION VIA SECOND-ORder Cone Programming RELAXATION}

Let us consider a WSN with $N$ anchors and one source, where the locations of the anchors are respectively denoted by $s_{1}, s_{2}, \ldots, s_{N}$, and the location of the unknown source is denoted by $\boldsymbol{x}$. Without loss of generality, this paper focuses on the 2-D scenario, i.e., $\boldsymbol{x}, \boldsymbol{s}_{1}, \boldsymbol{s}_{2}, \ldots, \boldsymbol{s}_{N} \in \mathbb{R}^{2}$ (the extension for a 3-D scenario is straightforward). For the sake of simplicity, we assume that all anchors are equipped with omnidirectional antennas and connected to the source. Furthermore, it is assumed that the anchor positions are known. Under the lognormal shadowing and log-distance path loss model, the path loss between the $i$ th anchor and the unknown source, i.e., $L_{i}$, can be modeled according to the following radio propagation path loss model (in decibels) [22]-[25], i.e.,

$$
L_{i}=L_{0}+10 \gamma \log _{10} \frac{\left\|\boldsymbol{x}-\boldsymbol{s}_{i}\right\|}{d_{0}}+v_{i}, \quad i=1, \ldots, N
$$

where $L_{0}$ denotes the path loss value at a short reference distance $d_{0}\left(\left\|\boldsymbol{x}-s_{i}\right\| \geq d_{0}\right), \gamma$ is the PLE, and $v_{i}$ is the lognormal shadowing term modeled as a zero-mean Gaussian random variable with variance $\sigma_{i}^{2}$, i.e., $v_{i} \sim \mathcal{N}\left(0, \sigma_{i}^{2}\right)$. The model has been validated by a variety of measurement results [23]-[28].

From the relationship $L_{i}(\mathrm{~dB})=10 \log _{10}\left(P_{T} / P_{i}\right)$, where $P_{i}$ is the RSS measured by the $i$ th anchor, and $P_{T}$ is the transmission power of the unknown source, it is easy to see that the localization problem can be formulated by the path loss instead of the RSS. Hence, as in [12] and [13], the pathloss-based approach is adopted in this paper. Based on the measurements in (1), the ML estimator is found by solving the nonlinear and nonconvex LS problem, i.e.,

$$
\hat{\boldsymbol{x}}=\underset{\boldsymbol{x}}{\arg \min } \sum_{i=1}^{N} \frac{1}{\sigma_{i}^{2}}\left[\left(L_{i}-L_{0}\right)-10 \gamma \log _{10} \frac{\left\|\boldsymbol{x}-\boldsymbol{s}_{i}\right\|}{d_{0}}\right]^{2} .
$$

To solve (2), recursive methods such as Newton's method, combined with the gradient descent method, are often used [19]. However, the objective function may have many local optima, and local search methods may easily get trapped in a local optimum. Hence, in this paper, we employ convex relaxation to address the nonconvexity of the localization problem.

The remainder of this section is organized as follows. Section II-A deals with the case where $P_{T}$ is known, whereas Section II-B deals with the case where $P_{T}$ is considered to be an unknown parameter that needs to be estimated. Finally, Section II-C addresses the more general problem when $P_{T}$ and PLE are simultaneously unknown.

\section{A. Noncooperative Scenario With Known $P_{T}$}

The source might be designed to measure and report its own calibration data to the anchors, in which case it is reasonable to assume that the source transmission power is known [5]. This corresponds to the case when the reference path loss $L_{0}$, which depends on $P_{T}$ [22], is known. 
For the sake of simplicity, in the rest of this paper, we assume that $\sigma_{i}^{2}=\sigma^{2}$, for $i=1, \ldots, N$. When the noise is sufficiently small, from (1), we get

$$
\alpha_{i}\left\|\boldsymbol{x}-\boldsymbol{s}_{i}\right\| \approx d_{0}
$$

where $\alpha_{i}=10^{\left(L_{0}-L_{i}\right) / 10 \gamma}$. One way for estimating the source location $\boldsymbol{x}$ is via the minimization of the LS criterion. Thus, according to (3), the LS estimation problem can be formulated as ${ }^{1}$

$$
\hat{\boldsymbol{x}}=\underset{\boldsymbol{x}}{\arg \min } \sum_{i=1}^{N}\left(\alpha_{i}\left\|\boldsymbol{x}-\boldsymbol{s}_{i}\right\|-d_{0}\right)^{2} .
$$

Although the problem in (4) is nonconvex, when $\alpha_{i}=d_{0}$, for $i=1, \ldots, N$, it can be accurately solved by the SCLP method presented in [30]. ${ }^{2}$ In the following text, we will present a novel approach to solve the problem defined in (4).

Defining auxiliary variables $\boldsymbol{z}=\left[z_{1}, \ldots, z_{N}\right]^{T}$, where $z_{i}=$ $\alpha_{i} g_{i}-d_{0}$, and $g_{i}=\left\|\boldsymbol{x}-\boldsymbol{s}_{i}\right\|$, from (4), we get

$$
\underset{\boldsymbol{x}, \boldsymbol{g}, \boldsymbol{z}}{\operatorname{minimize}}\|\boldsymbol{z}\|^{2}
$$

subject to

$$
g_{i}=\left\|\boldsymbol{x}-\boldsymbol{s}_{i}\right\|, z_{i}=\alpha_{i} g_{i}-d_{0}, i=1, \ldots, N .
$$

Introducing an epigraph variable $t$ and relaxing the nonconvex constraint $g_{i}=\left\|\boldsymbol{x}-\boldsymbol{s}_{i}\right\|$ as $g_{i} \geq\left\|\boldsymbol{x}-\boldsymbol{s}_{i}\right\|$ yield the following SOCP problem:

$$
\underset{\boldsymbol{x}, \boldsymbol{g}, \boldsymbol{z}, t}{\operatorname{minimize}} t
$$

subject to

$$
\begin{aligned}
\|[2 z ; t-1]\| & \leq t+1,\left\|\boldsymbol{x}-\boldsymbol{s}_{i}\right\| \leq g_{i}, \\
z_{i} & =\alpha_{i} g_{i}-d_{0}, i=1, \ldots, N .
\end{aligned}
$$

Problem (6) can be efficiently solved by [21], and we will refer to it as "SOCP1" in the following text. ${ }^{3}$

\section{B. Noncooperative Scenario With Unknown $P_{T}$}

The assumption that the anchors know the actual source transmission power may be too strong in practice since it would require additional hardware in both source and anchors [5].

\footnotetext{
${ }^{1} \mathrm{~A}$ justification for dropping the shadowing term in the propagation path loss model is provided in the following text. We can rewrite (1) as $\left(L_{i}-L_{0}\right) / 10 \gamma=\log _{10}\left(\left\|\boldsymbol{x}-\boldsymbol{s}_{i}\right\| / d_{0}\right)+\left(v_{i} / 10 \gamma\right)$, which corresponds to $\alpha_{i}\left\|\boldsymbol{x}-\boldsymbol{s}_{i}\right\|=d_{0} 10^{v_{i} / 10 \gamma}$. For sufficiently small noise, the first-order Taylor series expansion to the right-hand side of the previous expression is given by $\alpha_{i}\left\|\boldsymbol{x}-\boldsymbol{s}_{i}\right\|=d_{0}\left(1+(\ln 10 / 10 \gamma) v_{i}\right)$, i.e., $\alpha_{i}\left\|\boldsymbol{x}-\boldsymbol{s}_{i}\right\|=d_{0}+\epsilon_{i}$, where $\epsilon_{i}=d_{0}(\ln 10 / 10 \gamma) v_{i}$ is the zero-mean Gaussian random variable with the variance $d_{0}^{2}\left((\ln 10)^{2} / 100 \gamma^{2}\right) \sigma^{2}$. Clearly, the corresponding LS estimator is given by (4). The same has been done in [18].

${ }^{2}$ It is possible to generalize the SCLP method to the weighted case, i.e., to the case when $\alpha_{i} \neq d_{0}$ for some $i$. However, the algorithm in [30] yields a meaningless solution. This is due to the fact that the almost convexity property of the resulting constraints is not preserved.

"It is worth noting that the "SOCP1" approach can be modified to solve the localization problem in a distributed fashion.
}

Here, a more realistic and challenging scenario where the anchor nodes are not aware of the source transmission power is considered; thus, $L_{0}$ is assumed to be unknown and has to be estimated. The joint ML estimation of $\boldsymbol{x}$ and $L_{0}$ can be formulated as

$\hat{\boldsymbol{\theta}}=\underset{\boldsymbol{\theta}=\left[\boldsymbol{x} ; L_{0}\right]}{\arg \min } \sum_{i=1}^{N} \frac{1}{\sigma^{2}}\left[\left(L_{i}-\boldsymbol{l}^{T} \boldsymbol{\theta}\right)-10 \gamma \log _{10} \frac{\left\|\boldsymbol{A}^{T} \boldsymbol{\theta}-\boldsymbol{s}_{i}\right\|}{d_{0}}\right]^{2}$

where $\boldsymbol{l}=\left[\mathbf{0}_{2 \times 1} ; 1\right]$, and $\boldsymbol{A}=\left[\boldsymbol{I}_{2} ; \mathbf{0}_{1 \times 2}\right]$.

In (3), we assumed that $P_{T}$, i.e., $L_{0}$ is known. Assuming that $L_{0}$ is unknown, we can rewrite (3) as

$$
\psi_{i}\left\|\boldsymbol{x}-\boldsymbol{s}_{i}\right\| \approx \eta d_{0}
$$

where $\psi_{i}=10^{-L_{i} / 10 \gamma}$, and $\eta=10^{-L_{0} / 10 \gamma}$. By following a procedure similar to that in Section II-A, we obtain the SOCP problem, i.e.,

$$
\operatorname{minimize}_{\boldsymbol{x}, \boldsymbol{z}, \eta} t
$$

subject to

$$
\begin{aligned}
\|[2 \boldsymbol{z} ; t-1]\| & \leq t+1,\left\|\boldsymbol{x}-\boldsymbol{s}_{i}\right\| \leq g_{i} \\
z_{i} & =\psi_{i} g_{i}-\eta d_{0}, i=1, \ldots, N .
\end{aligned}
$$

Although the approach in (9) efficiently solves (7), we can further improve its performance. To do so, we will exploit the estimate of $L_{0}$, i.e., $\widehat{L}_{0}$, which we get by solving (9), and solve another SOCP problem. This SOCP approach will be described in the following text.

Introducing auxiliary variables $r_{i}=\left\|\boldsymbol{x}-\boldsymbol{s}_{i}\right\|$ and $\gamma_{i}=r_{i}^{2}$, expanding (4), and dropping the term $d_{0}^{2}$, which has no effect on the minimization, yield

$$
\begin{array}{cl}
\underset{\boldsymbol{x}, \boldsymbol{\gamma}, \boldsymbol{r}}{\operatorname{minimize}} & \sum_{i=1}^{N}\left(\widehat{\alpha}_{i}^{2} \gamma_{i}-2 d_{0} \widehat{\alpha}_{i} r_{i}\right) \\
\text { subject to } \gamma_{i}=r_{i}^{2}, & r_{i}=\left\|\boldsymbol{x}-\boldsymbol{s}_{i}\right\|, i=1, \ldots, N
\end{array}
$$

where $\widehat{\alpha}_{i}=10^{\left(L_{i}-\widehat{L}_{0}\right) / 10 \gamma}$. One can relax (10) to a convex optimization problem as follows. The nonconvex constraint $\gamma_{i}=r_{i}^{2}$ will be replaced by the second-order cone constraint (SOCC) $r_{i}^{2} \leq \gamma_{i}$. In fact, the inequality constraint $r_{i}^{2} \leq \gamma_{i}$ will be satisfied as an equality since $\gamma_{i}$ and $r_{i}$ will decrease and increase in the minimization, respectively. Furthermore, define an auxiliary variable $y=\|\boldsymbol{x}\|^{2}$. The constraint $y=\|\boldsymbol{x}\|^{2}$ is relaxed to a convex constraint $y \geq\|\boldsymbol{x}\|^{2}$, which is evidently an SOCC. With the use of all developed constraints, problem (10) is approximated as a convex, SOCP, optimization problem, i.e.,

$$
\underset{\boldsymbol{x}, \boldsymbol{\gamma}, \boldsymbol{r}, y}{\operatorname{minimize}} \sum_{i=1}^{N}\left(\widehat{\alpha}_{i}^{2} \gamma_{i}-2 d_{0} \widehat{\alpha}_{i} r_{i}\right)
$$

subject to

$$
\begin{aligned}
\|[2 \boldsymbol{x} ; y-1]\| & \leq y+1,\left\|\left[2 r_{i} ; \gamma_{i}-1\right]\right\| \leq \gamma_{i}+1 \\
\gamma_{i} & =y-2 \boldsymbol{s}_{i}^{T} \boldsymbol{x}+\left\|\boldsymbol{s}_{i}\right\|^{2}, i=1, \ldots, N .
\end{aligned}
$$

